\title{
Improved Layout Structure with Complexity Measures for the Manufacturer's Pallet Loading Problem (MPLP) Using a Block Approach
}

\author{
Deemah M. Aljuhani (iD), Lazaros G. Papageorgiou (iD \\ Centre for Process Systems Engineering, Department of Chemical Engineering, UCL (University College London) (United Kingdom) \\ deemah.aljubani.16@ucl.ac.uk,l.Papageorgion@ucl.ac.uk
}

Received: July 2020

Accepted: October 2020

\begin{abstract}
:
Purpose: The purpose of this paper is to study the Manufacturers pallet-loading problem (MPLP), by loading identical small boxes onto a rectangle pallet to maximise the pallet utilization percentage while reducing the Complexity of loading.

Design/methodology/approach: In this research a Block approach is proposed using a Mixed integer linear programming (MILP) model that generates layouts of an improved structure, which is very effective due to its properties in grouping boxes in a certain orientation along the $\mathrm{X}$ and $\mathrm{Y}$ axis. Also, a novel complexity index is introduced to compare the complexity for different pallet loading, which have the same pallet size but different box arrangements.
\end{abstract}

Findings: The proposed algorithm has been tested against available data-sets in literature and the complexity measure and graphical layout results clearly demonstrate the superiority of the proposed approach compared with literature Manufacturers pallet-loading problem layouts.

Originality/value: This study aids real life manufactures operations when less complex operations are essential to reduce the complexity of pallet loading.

Keywords: manufactures pallet loading problems, cutting and packing problems, mathematical programming, mixed integer optimization

\section{To cite this article:}

Aljuhani, D.M., \& Papageorgiou, L.G. (2021). Improved layout structure with complexity measures for the Manufacturer's Pallet Loading Problem (MPLP) using a block approach. Journal of Industrial Engineering and Management, 14(2), 231-249. https://doi.org/10.3926/jiem.3264

\section{Introduction}

Pallet configuration is a critical function for efficient supply chains, as if effectively operated it will lead to a reduction in unnecessary operating costs including but not limited to; loading, storage, transportation, and workforce and will successfully drive customer service satisfaction. The problem has wide applications in packing, cargo, transportation, warehouse management and is commonly known as cutting and packing, knapsack problems, strip packing and bin packing.

In most of the literature, researchers refer to packing, cutting or packing and cutting problems as pallet loading problems due to the fact that cutting problems tend to find an optimal way to cut large objects into smaller ones, whereas packing problems place small objects optimally to form a larger object. All the problems have similar 
structure defining 2 sets of objects: "Large" and "Small". "Large" objects or Bins refer to the container, pallet or any other medium that would be used as a base of loading and configuration. "Small" items usually refer to boxes or products that would be packed into the Bins. (Dyckhoff, 1990) The Bins could be homogeneous or heterogeneous depending whether identical or various types of boxes or products are packed (Scheithauer \& Terno, 1996a):

- Manufacturer's Pallet Loading Problem (MPLP): boxes required for loading into the pallet are homogeneous

- Distributor's Pallet Loading Problem (DPLP): boxes required for loading into the pallet are heterogeneous

Usually, all problems in this research area have the following key constraints in common:

- $\quad$ Boxes edges must be parallel to the bin borders

- All boxes must be packed within the bin dimensions

- Boxes must not overlap with one-another

There are other constraints that could be considered depending on the problem at hand such as box orientation, weight of the cargo, stability, weight distribution, health and safety aspects such as; no mixing between pharmaceutical and detergents in a single pallet.

In the area of cutting and packing (C\&P) problems described by (Wäscher,Haußner \& Schumann, 2007) the MPLP problems can be categorized as a 2-Dimensional, rectangular, identical item packing problem known as (IIPP), where one layer solution is found then repeated to reach the manufacturers required height. Such IIPP are considered as pure layout problems regarding arranging small identical items. The only difference between pallet loading problems PLP and the cutting and packing problems is that no decisions on which products or items to be loaded is made. In this work we investigate the first type of problems (MPLP); related to the manufactures side, where identical boxes are packed into pallets of the same size and usually stored in the manufacturer's warehouse until shipping. We will investigate the problem from a two-dimensional (IIPP) aspect considering the graphical layout structure of the proposed Block model and comparing it to available graphical layouts from published research.

To successfully configure the pallets, mathematical models are needed; they would find the best placement of products and aim to maximize the number of boxes or minimize the space wastage between the boxes during loading. Researchers have considered solving such problems using two main approaches; either mathematical optimization models or heuristic approaches. Mathematical models are useful when pallet and box sizes are pre-specified and available. One of the common mathematical tools used in literature to solve such problems is linear programming, such as Tsai, Malstrom and Kuo (1993) and Arghavani and Abdou (1996).

The same concept applies to heuristic methods, where different criteria and methods are used to reach feasible solutions as in Ortmann, Ntene and Van Vuuren (2010) and Wei, Zhang and Chen (2009). Exact algorithms have been studied as the 01 mathematical model proposed by Beasley (1985) and Dowsland (1987); they used a graph theory to solve such problems. In 1988, (Bhattacharya, Roy \& Bhattacharya, 1998) proposed the maximal breadth filling sequence depth-first algorithm. A branch-and-cut algorithm was proposed by Alvarez-Valdés, Parreño and Tamarit (2005), later Lau, Chan, Tsui, Ho and Choy (2009) tackled the multi-pallet loading operations differently using a hybrid approach. In 2007; (Martins \& Dell, 2007) generated 3,080,730 different instances for the pallet loading problems that satisfy the area ratio of less than 101 boxes.

In 2009; Martins and Dell (2008) demonstrated an HVZ coding; representing the horizontal, vertical and zero box, respectively using an exact algorithm. A staircase structure has been proposed by Ji and Jin (2009) using a best first branch and bound algorithm. Castro and Oliveira (2011) proposed a formulation based on a hybrid discrete and continuous-space, inspired by scheduling models. In a similar approach of time representation, Castro and Grossmann (2012) proposed a new hybrid discrete and continuous-space formulation which was able to find and prove more optimal solutions than the previous DCS proposed by Castro and Oliveira (2011).

Block heuristics have also been considered and developed in the area of pallet loading, including G4- and G5- structures as in Lim, Ma, Xu and Zhang (2012) and Scheithauer and Terno (1996b). Ji and Jin (2009) proposed a 5 block 
heuristics for a larger scaled pallet loading problems, a HONG approach known as high-order non-guillotine was proposed by Martins and Dell (2008), which takes into account more than 5 block structures. Although block heuristics have proved in solving most data-sets to near optimal solutions, still more complex examples struggle to reach an optimal or near optimal solution. Lins, Lins and Morabito (2003) and Birgin, Lobato and Morabito (2010) proposed an L-shaped structure, where a recursive approach has been used and they assume that optimum solutions for all test problems have been found.

It is worth mentioning that although all the methods have promising efficiency in solving such problems, still many limitations to real life problems occur; such as the difficulty in running large packing problems instances, some specific dimension boxes or huge computational time to achieve acceptable and efficient results. Or they tend to provide more complex graphical layouts that require experienced and skill labor in additional to increased loading and packing time. In a summary the pallet configuration and loading problems have been widely studied over the past by various researchers due to the fact that the application in this area is extremely useful. Different solution methods ranging from and combining exact algorithms and heuristics have been explored by various researchers.

In this paper, we propose a novel mixed integer linear programming (MILP) model that generates layouts of an improved structure based on the Block representation. Where each Block groups boxes with the same orientation along the $\mathrm{X}$ and $\mathrm{Y}$ axis. The proposed optimisation-based approach has been tested against available literature datasets with supported graphical layout structures. The computational and graphical layout results show the superiority of the proposed approach compared with existing Manufacturers pallet-loading problem layouts. Up to our knowledge this is the first paper testing the Block approach using a mathematical linear model, as all previous Block approaches have been tested using heuristic algorithms.

In the following parts of this paper; the Materials and Methods section describes the detailed problem statement; followed by the mathematical formulation; the Results and Discussion section explores the comparison between the proposed method and existing literature; finally, the Conclusions section highlights findings and concluding remarks.

\section{Problem Definition}

Warehouses are limited to many constraints when it comes to pallet configuration such as pallet size, racks height and any costumer special configuration adjustment requirements before shipping. The aim is to configure the pallet in a way that it holds the maximum number of weekly homogeneous (identical) boxes immediately after the product is ready from the production lines. Usually various pallet dimensions, depending on the demand for shipping are used. Throughout this paper the following assumptions are made:

- All the boxes and pallets used assumed to be of rectangular shape

- All boxes have the option to rotate in a 2D, as shown in Figure 1

- Boxes cannot overlap with each other

- One layer of pallet layout is generated in $2 \mathrm{D}$ and could be repeated to reach the pallet height required by manufactures

Overall, the proposed problem can be stated as follows:

Given:

- Box dimensions in length $(\zeta$ and width (w)

- Pallet dimension $\left(X^{\max }, Y^{\max }\right)$

- Maximum number of Blocks allowed $(i, j)$

To determine:

- The number of individual boxes forming each Block with their selected orientation

- The size of each Block associated with its exact geometrical location 

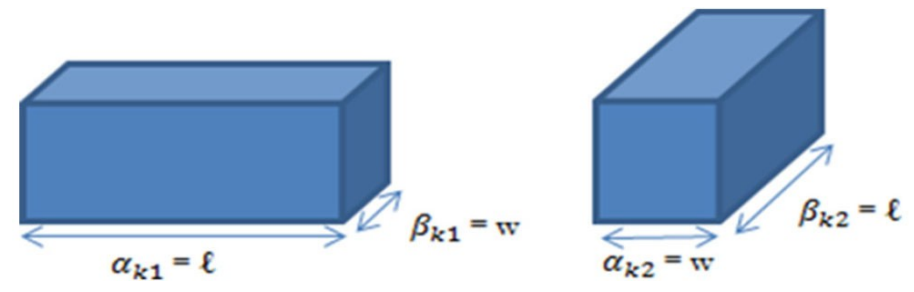

Figure 1. 2-Dimensional Box orientations

\section{Mathematical Model}

The pallet configuration problem can be defined as single sized boxes with a given dimension and the boxes can rotate in $2 \mathrm{D}$ defined as $\alpha_{\mathrm{k}}$ and $\beta_{\mathrm{k}}$ where $\mathrm{k}$ is the number of possible orientations either $\mathrm{k}=1$ or $\mathrm{k}=2$ as in Figure and Table 1. These boxes need to be configured and packed orthogonally within the pallet through Blocks known as $\mathrm{i}$ and $\mathrm{j}$, each Block shares the same individual boxes orientation. These Blocks are configured into a pallet with sizes defined as $X^{\max }$ and $Y^{\max }$ for the pallet length and width, respectively. Figure 2 illustrates the Block approach, where we have 5 Blocks; each is formed with several individual boxes of the same orientation. The detailed mathematical formulation of the model will follow up next.

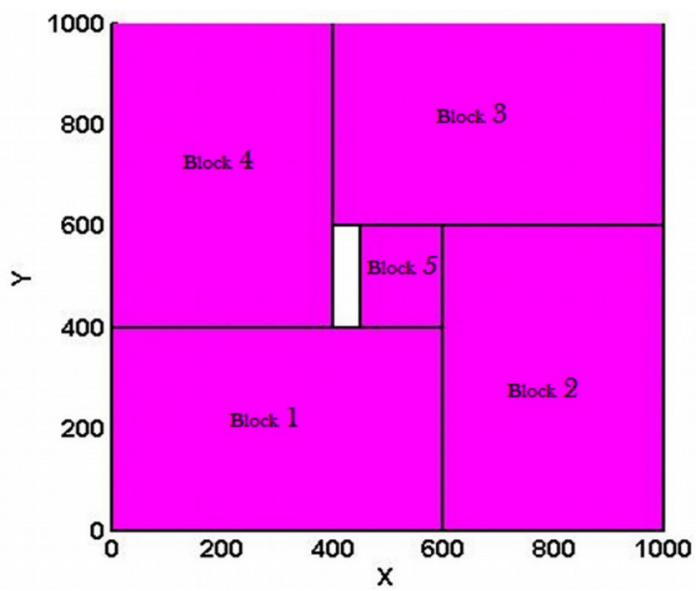

Figure 2. Block illustration

\begin{tabular}{|c|c|c|}
\hline & $\alpha$ & $\beta$ \\
\hline$K 1$ & 1 & $w$ \\
\hline$K 2$ & $w$ & 1 \\
\hline
\end{tabular}

Table 1. Possible box orientations

The indices and parameters associated with the Block approach are listed below:

\section{Indices}

$\begin{array}{ll}i, j & \text { Blocks } \\ k & \text { Possible orientations } \\ r, \bar{r} & \text { Boxes forming Blocks }\end{array}$

\section{Parameters}

$X^{\max }, Y^{\max }$ Pallet dimension in Length and Width

$\alpha_{\mathrm{k}}, \beta_{\mathrm{k}} \quad$ Box dimension

$U, M \quad$ Appropriate upper-bound value

UB Upper-bound on the objective function 
$\varepsilon \quad$ Penalty, a relativity small number

The formulation is based on the following key variables:

\section{Binary variables}

$Y_{\mathrm{ik}} \quad 1$ if orientation $\mathrm{k}$ is used for Block i; 0 otherwise

$w w_{\mathrm{i}} \quad$ Penalty binary variable

$Z L_{\text {ir }} \quad$ r boxes along the $\mathrm{X}$ axis

$Z W_{\text {ir }} \quad r$ boxes along the $\mathrm{Y}$ axis

$E 1_{i j}, E 2_{i j} \quad$ Non overlapping binary

$T_{i r} \quad$ Auxiliary variable $T_{i r}$

\section{Positive Variables}

BL Total length of Block i

$B W_{i} \quad$ Total width of Block $\mathrm{i}$

$S L_{i k} \quad$ Number of boxes in the $\mathrm{X}$ axis for each orientation $\mathrm{k}$ forming Block $\mathrm{i}$

$S W_{i k} \quad$ Number of boxes in the $\mathrm{Y}$ axis for each orientation $\mathrm{k}$ forming Block $\mathrm{i}$

$X_{i}, Y_{i} \quad$ Coordinates of geometrical center of Block $i$

$B_{i} \quad$ Number of individual boxes forming each Block $i$

\section{Integer Variables}

$N L_{i} \quad$ Number of boxes across the $\mathrm{X}$ axis forming Block $\mathrm{i}$

$N W_{i} \quad$ Number of boxes across the $\mathrm{Y}$ axis forming Block $\mathrm{i}$

\subsection{Objective Function}

The objective as mentioned earlier, is to maximize the utilization percentage of the pallet and that is done through maximizing the number of individual boxes $\mathrm{r}$ in each Block $i$ and $j$. We also apply a penalty $\varepsilon$ which is a relativity small number set to 0.001 here; to ensure we maximize the number of boxes used in each Block but at the same time minimize the number of Blocks used to adhere with the complexity measures we will go through later in the paper.

$$
\max \Sigma_{i} B_{i}-\varepsilon^{*} \sum_{i} w w_{i}
$$

\subsection{Constraints}

The number of boxes in each Block $\mathrm{i}$ is determined by selecting the appropriate combination of cardinality $r * \bar{r}$ using the Auxiliary binary variable $T_{i r}$ that is equal to 1 if that combination of block size is chosen and is zero otherwise; as the following in equation 2:

$$
B_{i}=\sum_{r \bar{r}} r^{*} \bar{r} * T_{i r \bar{r}} \forall i
$$

An ordering constraint for Blocks to be used in a sequence one after the other has been introducing as in equation 3:

$$
B_{i} \geq B_{i+1} \quad \forall i
$$

Length Orientation:

$$
\begin{gathered}
N L_{i}=\sum_{k} S L_{i k} \quad \forall i \\
N L_{i}=\sum_{r} r^{*} Z L_{i r} \quad \forall i \\
S L_{i k} \leq U^{*} Y_{i k} \quad \forall i, k \\
B L_{i}=\sum_{k} \alpha_{k}^{*} S L_{i k} \quad \forall i
\end{gathered}
$$

Equation 4 and 5 calculate the number of individual boxes across the $\mathrm{X}$ axis for each Block $i$. Equation 6 ensures that a length is calculated only if binary variable $Y_{i k}$ is active and its bounded by an upper bound $U$ which is set to 
the pallet Length. Finally, equation 7 calculates the total length used for each Block $i$, which is the box length selected $\alpha_{k}$ multiplied by the number of individual boxes selected in $S L_{i k}$.

\section{Width Orientation:}

In a similar approach the width is controlled:

$$
\begin{gathered}
N W_{i}=\sum_{k} S W_{i k} \quad \forall i \\
N W_{i}=\sum_{r} r^{*} Z W_{i r} \quad \forall i \\
S W_{i k} \leq U^{*} Y_{i k} \quad \forall i, k \\
B W_{i}=\sum_{k} \beta_{k} * S W_{i k} \quad \forall i
\end{gathered}
$$

\section{Lower bound constraints:}

Lower bound constraints on the coordinates of the geometrical center of each Block $i$ and $j$ have been considered to avoid intersection of blocks with the origin of axis as in the equations 12 and 13

$$
\begin{aligned}
& X_{i} \geq \frac{B L_{i}}{2} \quad \forall i \\
& Y_{i} \geq \frac{B W_{i}}{2} \quad \forall i
\end{aligned}
$$

Upper bound constraints:

In a similar way, upper bound constraints force the Blocks to be allocated within the pallet dimensions and the rectangular space is defined by the corners $(0,0)$ of the pallet dimensions $X^{\max }, Y^{\max }$ as the following:

$$
\begin{array}{ll}
X_{i}+\frac{B L_{i}}{2} \leq X^{\max } \quad \forall i \\
Y_{i}+\frac{B W_{i}}{2} \leq Y^{\max } \quad \forall i
\end{array}
$$

\section{Non-overlapping Constraints:}

To avoid Blocks $i$ and $j$ overlapping or occupying the same location in the $X$ and $Y$ axis we have introduced $E 1_{i j}$ and $E 2_{i j}$ binary variables as in (Papageorgiou \& Rotstein, 1998), The upper-bound Big $M$ value used here is equal to the pallet dimension in the Length $X^{\max }$ and it changes depending on the problem size.

Non overlapping in the $\mathrm{X}$ direction:

$$
\begin{gathered}
X_{i}-X_{j}+M\left(E 1_{i j}+E 2_{i j}\right) \geq \frac{B L_{i}+B L_{j}}{2} \quad \forall j \geq i \\
X_{j}-X_{i}+M\left(1-E 1_{i j}+E 2_{i j}\right) \geq \frac{B L_{i}+B L_{j}}{2} \quad \forall j \geq i
\end{gathered}
$$

Non overlapping in the Y direction:

$$
\begin{gathered}
Y_{i}-Y_{j}+M\left(1+E 1_{i j}-E 2_{i j}\right) \geq \frac{B W_{i}+B W_{j}}{2} \quad \forall j \geq i \\
Y_{j}-Y_{i}+M\left(2-E 1_{i j}-E 2_{i j}\right) \geq \frac{B W_{i}+B W_{j}}{2} \quad \forall j \geq i
\end{gathered}
$$




\subsection{Additional Design Constraints}

The number of boxes used in each direction has to be less than or equal to the maximum number of boxes that could be placed in that direction as in equation 20 and 21:

$$
\begin{array}{cc}
T_{i r \bar{r}} \leq Z L_{i r} & \forall i, r, \bar{r} \\
T_{i r \bar{r}} \leq Z W_{i \bar{r}} & \forall i, r, \bar{r}
\end{array}
$$

To apply the penalty to the objective function in equation 1 we need to ensure that the binary variable $w w_{i}$ is active only when $Y_{i k}$ is active as in equation 22, and to ensure boxes are only assigned to Blocks, if that Block has been selected, equations 23 and 24 have been added:

$$
\begin{array}{cc}
\sum_{k} y_{i k}=w w_{i} & \forall i \\
\sum_{r} Z L_{i r}=w w_{i} & \forall i \\
\sum_{r} Z W_{i r}=w w_{i} & \forall i
\end{array}
$$

Due to the geometry of the Block approach, alternative symmetrical layout solutions could be received when equivalent optimal solutions exist, which requires longer CPU times. So in order to have a better efficiency in terms of the CPU time the symmetry-breaking constraints are introduced as in (Westerlund \& Papageorgiou, 2004) by fixing the very first box to the "bottom left corner" in the following manner.

$$
\begin{gathered}
X_{1}=\frac{B L_{1}}{2} \\
Y_{1}=\frac{B W_{1}}{2} \\
B L_{1} \geq B W_{1}
\end{gathered}
$$

Finally, to reduce the computatonal effort an upperbound on the objective function value has been set to the maximum utilization pecentage for the pallet/box size as the following:

$$
\mathrm{UB}=\frac{\mathrm{X}^{\max } * \mathrm{Y}^{\max }}{\alpha_{\mathrm{k} 1} * \beta_{\mathrm{k} 1}}
$$

\section{Complexity Measure}

As stated before, the main objective of the proposed model is to assist with providing less complex graphical layouts of the pallet loading problem, and to assist with that a novel metric, here referred to as; the Complexity measure is introduced. The metric aids in comparing 2 pallet layouts that share the same pallet size $X^{\max }$ and $Y^{\max }$ as well as the same number of boxes loaded $B_{i}$ but generated using different mathematical approaches which as a result provide different box arrangements. The layout complexity measure can be described using the following mathematical formulation:

$$
\zeta=\frac{\text { Vrchange }+ \text { Hrchange }}{\left(2 \Sigma_{i} B_{i}\right)-x r-y c}
$$

Where; $\zeta$ captures the average number of orientation changes between the boxes in the pallet and it takes any value between 0 and 1 describing the complexity as the following:

- 0 indicates the least complex arrangement

- the most complex possible arrangement 
The Vrchange and Hrchange capture the Vertical and Horizontal orientation changes in the $\mathrm{X}$ and $\mathrm{Y}$ axis of the pallet, respectively. $B_{i}$ is the number of boxes loaded in the pallet and is generated from the MINLP model proposed above. The $x r$ and $y r$ represent the number of boxes in the 1 rst row and column along the $\mathrm{X}$ and $\mathrm{Y}$ axis, respectively, as seen in Figure 3.

To further demonstrate the concept of the (0-1) complexity metric values, assume a pallet containing boxes that share the same orientation; in this case the pallet will only have one Block and when the above metric is applied, the $V$ rchange and Hrchange will be equal to zero as no changes in the orientations are detected which will lead to a complexity index $\zeta=0$, indicating a simple arrangement. On the other hand, if a pallet is loaded in a way that each box has a different orientation than the adjacent box, the number of Blocks in the pallet will be equal to the number of individual boxes loaded and the number of Vrchange and Hrchange will be equal to 2 multiplied by the number of boxes loaded minus the number of boxes on the first row and column of the pallet ( $x r$ and $y r$ ) leading to a complexity index $\zeta=1$, making it a complex arrangement. Finally, the flow chart in Figure 4 demonstrates the calculation method.

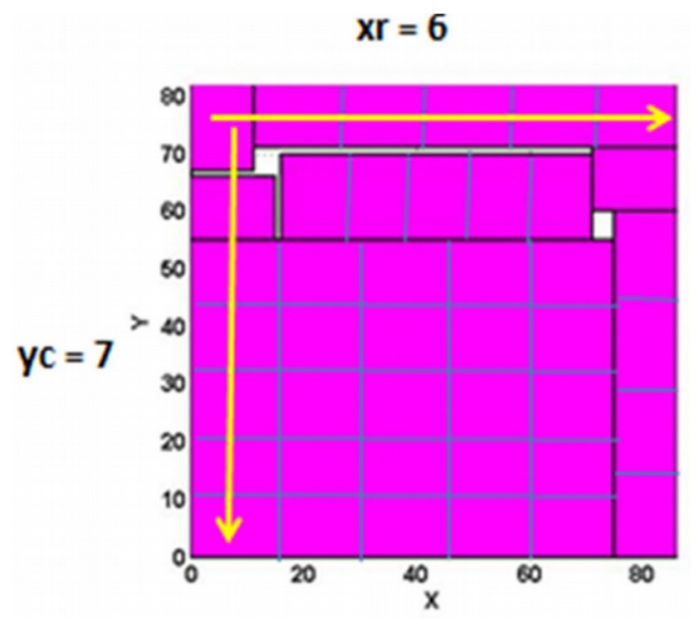

Figure 3. Calculation of $\mathrm{xr}$ and yc for Complexity Index $\zeta$

As seen in Figure 4, the calculations for the Vertical Vrchange and Horizontal Hrchange changes are made simultaneously; where at the first stage it is assumed that all boxes on the first row and column hold a value of zero, due to the fact that they are the starting points in the pallet and no rows or columns of boxes exist before them. As we move through the pallet vertically and horizontally, we check whether a change in orientation has been detected between each box and the previous box; if detected that box is given a value of 1 and if no change is seen the box is given a value of zero. This process is repeated and terminated once the total number of boxes $B_{i}$ in the pallet is reached. As a final step the total number of changes seen in the pallet is summed up and applied to the Complexity Measure equation 21 to find the value of $\zeta$. The above metric will be applied to different literature layouts and compared with the layouts generated from the Block approach described in this paper. 


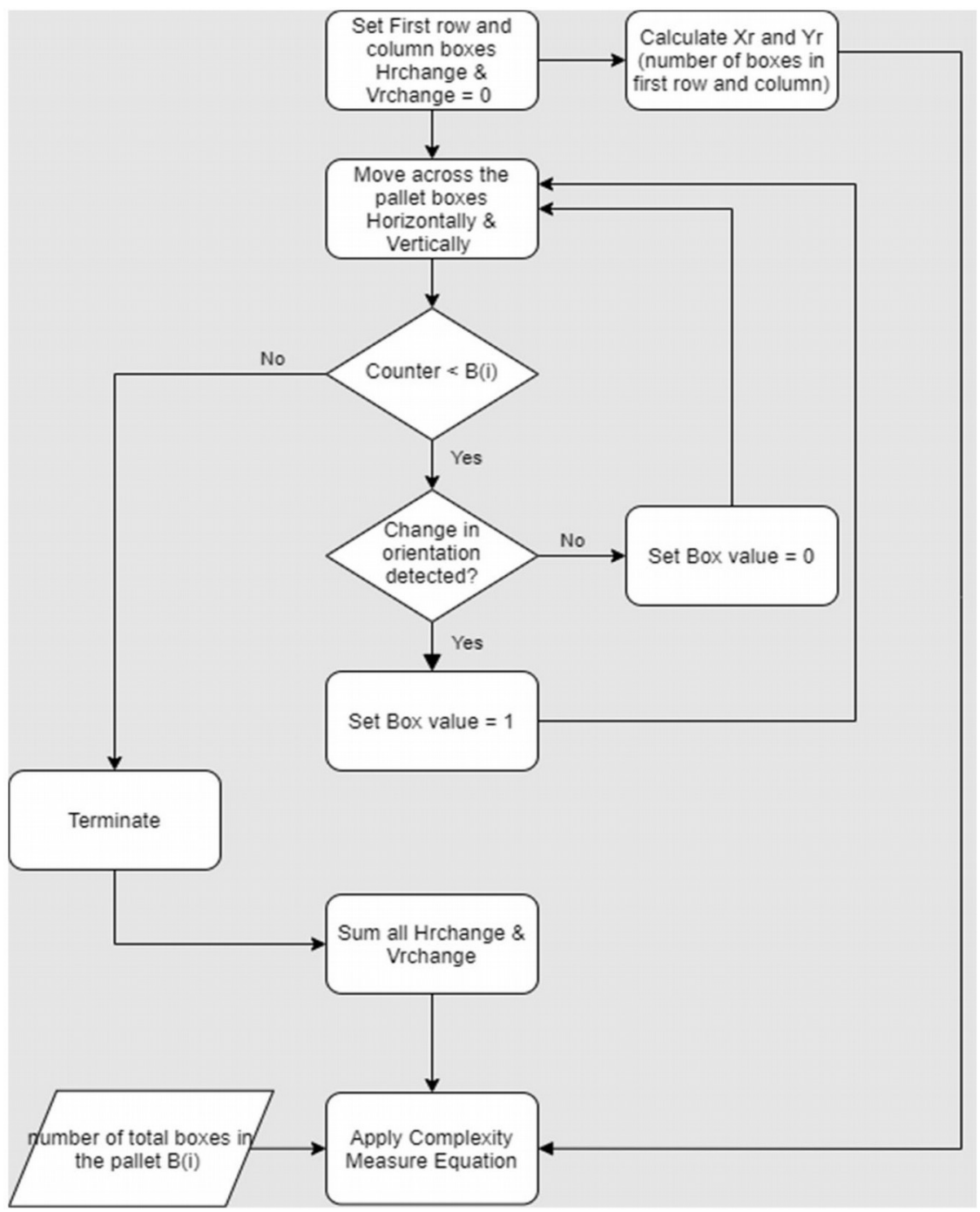

Figure 4. Complexity Measure flowchart

\section{Result and Discussion}

\subsection{Benchmark Instances}

To evaluate the performance of the proposed model it has been tested against the Benchmark instances reported in the pallet loading problems review paper by (Silva, Oliveira \& Waescher, 2016). A decision on selecting these instances for testing and validating the proposed Block model was made due to the fact that they cover all instances that have been listed and tested in at least two pallet loading experiments from literature since 1982 up to recent years and they clearly demonstrate a wide range of instances ranging from small, medium to large ones, they have been solved using exact and heuristic approaches. Instances (1-25) and (35-45) have been solved using both exact algorithms and heuristic approaches in literature while instances (26-34) and (46-55) have only been tested using heuristic approaches. We believe that testing against a wide range of datasets proves the validity of the proposed model in this paper. Table 2 represents the instances, the pallet dimensions $X^{\max }$ and $Y^{\max }$ the individual box dimension $\alpha$ and $\beta$, the objective value obtained from the published papers $z$, the results obtained from the Block approach Boxes and finally where the instances have been tested 


\begin{tabular}{|c|c|c|c|c|c|c|c|}
\hline$\#$ & $\boldsymbol{X}^{\max }$ & $Y_{\max }^{\max }$ & $\alpha$ & $\beta$ & $\mathrm{z}$ & Boxes & Papers where the instances were used \\
\hline 1 & 1000 & 1000 & 205 & 159 & 30 & 30 & \multirow{2}{*}{$\begin{array}{l}\text { Bischoff \& Dowsland, 1982; Wu \& Ting, 2007; Young-Gun \& } \\
\text { Kang, } 2001\end{array}$} \\
\hline 2 & 1000 & 1000 & 200 & 150 & 33 & 33 & \\
\hline 3 & 14 & 10 & 3 & 2 & 23 & 23 & Dowsland, 1984; Wu \& Ting, 2007; Young-Gun \& Kang, 2001 \\
\hline 4 & 16 & 11 & 3 & 2 & 29 & 29 & \multirow{2}{*}{$\begin{array}{l}\text { Arenales \& Morabito, 1995, Bhattacharya et al., 1998; } \\
\text { Dowsland, 1984; Martins \& Dell, 2008; Morabito \& Morales, } \\
\text { 1998; Pureza \& Morabito, 2003; Wu \& Ting, 2007; Young- } \\
\text { Gun \& Kang, 2001 }\end{array}$} \\
\hline 5 & 86 & 82 & 15 & 11 & 42 & 42 & \\
\hline 6 & 30 & 22 & 7 & 4 & 23 & 23 & \multirow{6}{*}{$\begin{array}{l}\text { Arenales \& Morabito, 1995; Bhattacharya et al., 1998; Wu \& } \\
\text { Ting, 2007; Young-Gun \& Kang, 2001; Arenales \& Morabito, } \\
\text { 1995; Bhattacharya et al., 1998; Dowsland, 1984; Wu \& Ting, } \\
\text { 2007; Young-Gun \& Kang, 2001 }\end{array}$} \\
\hline 7 & 46 & 34 & 11 & 6 & 23 & 23 & \\
\hline 8 & 50 & 36 & 11 & 7 & 23 & 23 & \\
\hline 9 & 53 & 51 & 9 & 7 & 42 & 42 & \\
\hline 10 & 63 & 60 & 11 & 8 & 42 & 42 & \\
\hline 11 & 76 & 73 & 13 & 10 & 42 & 42 & \\
\hline 12 & 87 & 47 & 7 & 6 & 97 & 97 & $\begin{array}{l}\text { Amaral \& Wright, 2001; Bhattacharya et al., 1998; Martins \& } \\
\text { Dell, 2008; Morabito \& Morales, 1998; Nelißen, 1995; Pureza } \\
\text { \& Morabito, 2003, 2006; Wu \& Ting, 2007; Young-Gun \& } \\
\text { Kang, } 2001\end{array}$ \\
\hline 13 & 57 & 44 & 12 & 5 & 41 & 41 & $\begin{array}{l}\text { Martins \& Dell, 2008; Morabito \& Morales, 1998; Nelißen, } \\
\text { 1995; Pureza \& Morabito, 2003, 2006; Scheithauer \& Terno, } \\
\text { 1996a; Wu \& Ting, 2007; Young-Gun \& Kang, } 2001\end{array}$ \\
\hline 14 & 40 & 33 & 7 & 4 & 46 & 46 & $\begin{array}{l}\text { Bhattacharya et al., 1998; Nelißen, 1995; Wu \& Ting, 2007; } \\
\text { Young-Gun \& Kang, 2001; Ribeiro \& Lorena, 2007; Letchford } \\
\text { \& Amaral, } 2001\end{array}$ \\
\hline 15 & 3750 & 3063 & 646 & 375 & 46 & 46 & \multirow{3}{*}{$\begin{array}{l}\text { Bhattacharya et al., 1998; Nelißen, 1995; Wu \& Ting, 2007; } \\
\text { Young-Gun \& Kang, } 2001\end{array}$} \\
\hline 16 & 1200 & 800 & 176 & 135 & 38 & 38 & \\
\hline 17 & 34 & 23 & 5 & 4 & 38 & 38 & \\
\hline 18 & 300 & 200 & 21 & 19 & 149 & 149 & $\begin{array}{l}\text { Martins \& Dell, 2008; Morabito \& Morales, 1998; Scheithauer } \\
\text { \& Terno, 1996a }\end{array}$ \\
\hline 19 & 40 & 25 & 7 & 3 & 47 & 47 & \multirow{3}{*}{$\begin{array}{l}\text { Martins \& Dell, 2008; Morabito \& Morales, 1998; Pureza \& } \\
\text { Morabito, 2003, 2006; Scheithauer \& Terno, 1996a; Wu \& } \\
\text { Ting, } 2007\end{array}$} \\
\hline 20 & 52 & 33 & 9 & 4 & 47 & 47 & \\
\hline 21 & 27 & 18 & 7 & 4 & 99 & 99 & \\
\hline 22 & 43 & 26 & 7 & 3 & 53 & 53 & \multirow{2}{*}{$\begin{array}{l}\text { Lins et al., 2003; Martins \& Dell, 2008; Morabito \& Morales, } \\
\text { 1998; Pureza \& Morabito, 2003, 2006; Wu \& Ting, 2007; } \\
\text { Birgin et al., } 2005\end{array}$} \\
\hline 23 & 153 & 100 & 24 & 7 & 90 & 90 & \\
\hline 24 & 42 & 39 & 9 & 4 & 45 & 45 & \multirow{2}{*}{$\begin{array}{l}\text { Martins \& Dell, 2008; Morabito \& Morales, 1998; Pureza \& } \\
\text { Morabito, 2003, 2006; Wu \& Ting, } 2007\end{array}$} \\
\hline 25 & 124 & 81 & 21 & 10 & 47 & 47 & \\
\hline 26 & 100 & 64 & 17 & 10 & 36 & 36 & \multirow[t]{9}{*}{ Ribeiro \& Lorena, 2007; Letchford \& Amaral, 2001} \\
\hline 27 & 100 & 82 & 22 & 8 & 45 & 45 & \\
\hline 28 & 100 & 83 & 22 & 8 & 45 & 45 & \\
\hline 29 & 32 & 22 & 5 & 4 & 34 & 34 & \\
\hline 30 & 32 & 27 & 5 & 4 & 42 & 42 & \\
\hline 31 & 40 & 26 & 7 & 4 & 36 & 36 & \\
\hline 32 & 53 & 26 & 7 & 4 & 48 & 48 & \\
\hline 33 & 37 & 30 & 8 & 3 & 45 & 45 & \\
\hline 34 & 81 & 39 & 9 & 7 & 49 & 49 & \\
\hline
\end{tabular}




\begin{tabular}{|c|c|c|c|c|c|c|c|}
\hline$\#$ & $\boldsymbol{X}^{\max }$ & $Y^{\max }$ & $\alpha$ & $\beta$ & $\mathrm{z}$ & Boxes & Papers where the instances were used \\
\hline 35 & 61 & 38 & 6 & 5 & 77 & 77 & $\begin{array}{l}\text { Lins et al., 2003; Martins \& Dell, 2008; Pureza \& Morabito, } \\
\text { 2003, 2006; Wu \& Ting, 2007; Ribeiro \& Lorena, 2007; Birgin, } \\
\text { Morabito \& Nishihara, } 2005\end{array}$ \\
\hline 36 & 63 & 44 & 8 & 5 & 69 & 69 & \multirow{7}{*}{$\begin{array}{l}\text { Lins et al., 2003; Martins \& Dell, 2008; Pureza \& Morabito, } \\
\text { 2003, 2006; Wu \& Ting, 2007; Birgin et al., } 2005\end{array}$} \\
\hline 37 & 61 & 35 & 10 & 3 & 71 & 71 & \\
\hline 38 & 61 & 38 & 10 & 3 & 77 & 77 & \\
\hline 39 & 93 & 46 & 13 & 4 & 82 & 82 & \\
\hline 40 & 106 & 59 & 13 & 5 & 96 & 96 & \\
\hline 41 & 141 & 71 & 13 & 8 & 96 & 96 & \\
\hline 42 & 108 & 65 & 10 & 7 & 100 & 100 & \\
\hline 43 & 86 & 52 & 9 & 5 & 99 & 99 & \multirow{2}{*}{$\begin{array}{l}\text { Alvarez-Valdés et al., 2005; Lins et al., 2003; Pureza \& } \\
\text { Morabito, 2003, 2006; Wu \& Ting, 2007; Birgin et al., } 2005\end{array}$} \\
\hline 44 & 74 & 46 & 7 & 5 & 97 & 97 & \\
\hline 45 & 67 & 44 & 6 & 5 & 97 & 97 & $\begin{array}{l}\text { Lins et al., 2003; Pureza \& Morabito, 2003, 2006; Wu \& Ting, } \\
2007\end{array}$ \\
\hline 46 & 49 & 28 & 8 & 3 & 57 & 57 & \multirow[t]{5}{*}{ Lins et al., 2003; Martins \& Dell, 2008; Birgin et al., 2005} \\
\hline 47 & 57 & 34 & 7 & 4 & 69 & 69 & \\
\hline 48 & 67 & 37 & 11 & 3 & 75 & 75 & \\
\hline 49 & 67 & 40 & 11 & 3 & 81 & 81 & \\
\hline 50 & 74 & 49 & 11 & 4 & 82 & 82 & \\
\hline 51 & 1600 & 1230 & 137 & 95 & 147 & 147 & \multirow[t]{5}{*}{ Birgin et al., 2010; Ribeiro \& Lorena, 2007} \\
\hline 52 & 2536 & 1312 & 144 & 84 & 273 & 273 & \\
\hline 53 & 2252 & 1470 & 144 & 84 & 271 & 271 & \\
\hline 54 & 1470 & 1458 & 144 & 84 & 175 & 175 & \\
\hline 55 & 2296 & 1230 & 135 & 92 & 226 & 226 & \\
\hline
\end{tabular}

Table 2. Literature Instance Data-Sets with Block Approach results (Silva et al., 2016)

The number of blocks $i, j$ in all instances has been set to 15 Blocks, where the proposed algorithm can freely choose the number of Blocks required and due to the penalty term added to the objective function the max number of Blocks seen in all instances tested has been 7 blocks. Also the value of the individual boxes forming the Blocks $r, \bar{r}$ has been set to the max number of boxes that could be placed across any axis of the pallet as the following: $\max (L, W) / \min (\alpha, \beta)$ and this number is different depending on the problem size.

It should be noted that all datasets have been solved using GAMS modelling system with CPLEX mixed-integer linear programming optimization package, on an Intel®Xeon®E5-1620 CPU with16GB RAM, the computing times ranged between 5 seconds for small instances up to a limit of 1000 seconds for more complex ones (average around 400s). Taking into account that such problems are classified as NP-hard, the computational times are considered within a reasonable computational effort range. In addition, it can be noted that the Block approach has obtained the same results as those reported in Literature in terms of number of boxes loaded onto pallets but with layouts of reduced complexity, as clearly shown in the next section.

\subsection{Block Approach Layouts}

The proposed algorithm in this paper and as previously mentioned is a similar structure compared to the idea of heuristic block methods, such as the 4-block pattern, the G4-structure and the 5 Block structure. The similarity arises in the idea of grouping boxes that share the same orientation in one Block. The proposed algorithm differs from heuristic approaches, apart from being an optimization-based model is the functionality of being an n-Block approach where the boxes are not forced in a certain predefined number of Blocks. The boxes are freely grouped 
into Blocks on the pallet dimension depending on the problem size. Up to our knowledge, the linear based approach for Block structures used in this paper has not been presented elsewhere and the novelty of the Complexity measure for layout comparisons is considered a new approach. The following section will be looking into the complexity measure with comparisons between the Block approach layouts and Literature layouts. A selection of three instances from Table 1 have been tested and compared; instance number 4 followed by instance 5,18 as they cover different size of complexity ranging from small, medium to large. Additional layout comparisons could be seen in Appendix A.

\subsection{Graphical Layout Comparison}

A comparison between the layout presented for instance 4 in Table 2 against the layout generated from the Block approach is displayed in Figure 5, it could be noted that the layout generated from the Block approach looks simpler in terms of block numbers, where the boxes are grouped into only 2 Blocks while literature layout presented in Young-Gun and Kang (2001) using a heuristic 5 Block approach has about 7 Blocks. Such simplification seen in the Block approach is a definite advantage that will help in supply chain costs reduction by reducing the loading times in both manual and robotic loading. We then apply the above Complexity metric represented in equation 27 to calculate the Complexity Index $\zeta$. The detailed steps for calculating the $V$ rchange and Hrchange (number of orientation changes) for each layout is presented in Figure 6, where we assign a value of 0 or 1 to each individual box whether it holds the same orientation as its adjunct previous neighbour box or not in the Vertical (Y) axis and Horizontal (X) axis; respectively. We then sum the number of changes for each individual box and apply the complexity metric equation to obtain the level of complexity for the layout, the final comparison results are shown in Table 3.

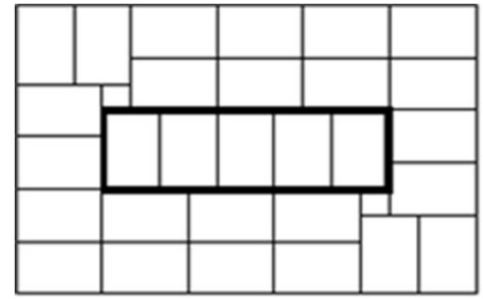

(a) Literature Layout (Young-Gun \& Kang, 2001)

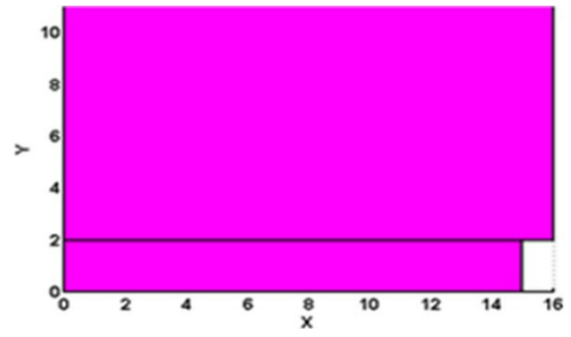

(b) Block Layout

Figure 5. Layouts of Instance 4

\begin{tabular}{|l|c|c|c|c|c|c|c|}
\hline Approach & VC & HC & $\boldsymbol{\Sigma} \boldsymbol{B}_{\mathbf{i}}$ & $\mathbf{x r}$ & yc & $\boldsymbol{\zeta}$ & Number of Blocks \\
\hline Block & 5 & 0 & 29 & 8 & 4 & 0.10 & 2 \\
\hline Literature (heuristic 5-Block approach) & 11 & 6 & 29 & 6 & 5 & 0.36 & 7 \\
\hline
\end{tabular}

Table 3. Complexity Index for Instance 4

As it can be seen from the above comparison, the complexity index $\zeta$ for the Block approach is 0.10 , which is closer to zero; resulting in a less complex box layout compared to literature complexity index $\zeta$ of 0.36 ie. more complex layout. It can also be noted that the number of rotations from one box to the other VRchanges and HRchanges is much less in the Block approach compared to literature, allowing the boxes that share the same rotation to be grouped, where possible. Another interesting comparison is the number of blocks used to construct the pallet, which is equal to 7 in the literature results but only 2 in the block approach.

In a similar approach, Instance 5 from Table 2 is investigated, Figure 7 represents the layout from literature presented in (Arenales \& Morabito, 1995) using a AND/OR-graph approach and the Block model. The method of Complexity again is displayed in Figure 8 and the Complexity Index comparison is displayed in Table 4. Where we can see a $\zeta$ value of 0.025 for the Block approach and a 0.33 for the literature. The number of blocks to construct the pallet is 6 and 8 for the block approach and literature; respectively. Although this difference in complexity index 
$\zeta$ and number of blocks used to construct the pallet might not seem high but when considering plants with thousands of pallets to load daily a significant time reduction would be seen.
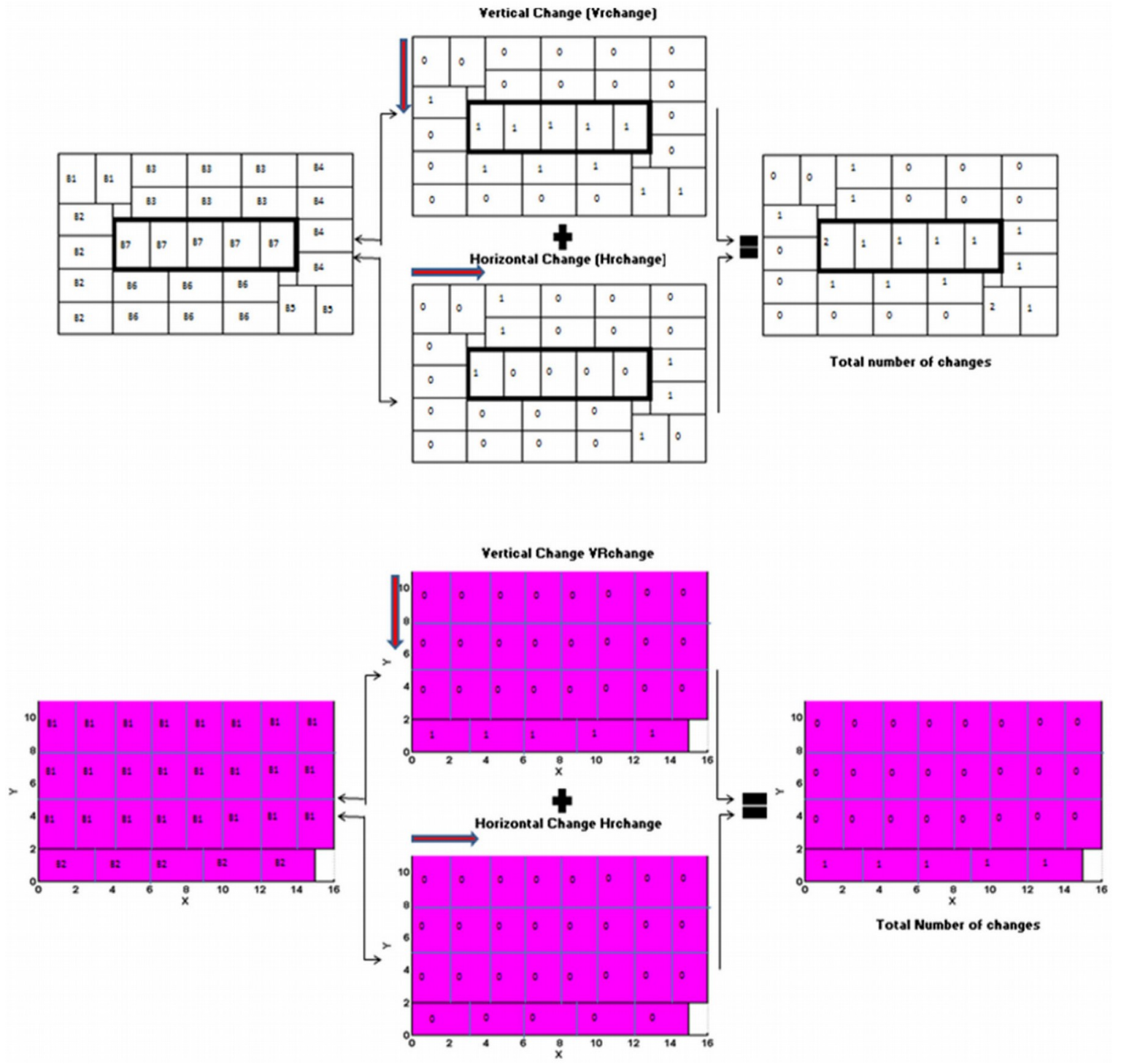

Figure 6. Complexity Index Calculation method for Instance 4

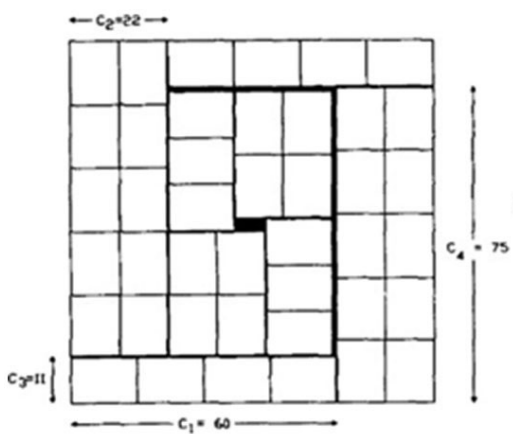

(a) Literature Layout (Arenales \& Morabito, 1995)

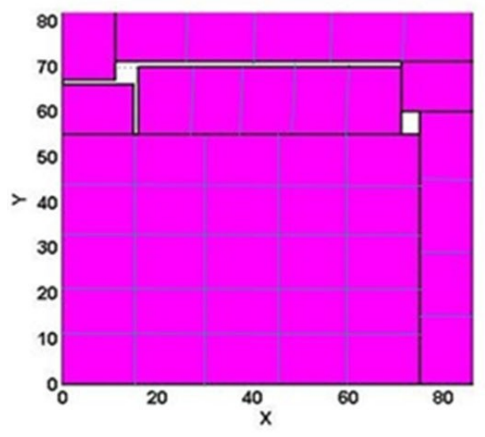

(b) Block Layout

Figure 7. Layouts of Instance 5 


\begin{tabular}{|l|c|c|c|c|c|c|c|}
\hline Approach & VC & HC & $\boldsymbol{\Sigma} \boldsymbol{B}_{\mathrm{i}}$ & $\mathbf{x r}$ & yc & $\boldsymbol{\zeta}$ & Number of Blocks \\
\hline Block & 11 & 7 & 42 & 6 & 7 & 0.25 & 7 \\
\hline Literature (AND/OR-graph approach) & 12 & 12 & 42 & 6 & 6 & 0.33 & 8 \\
\hline
\end{tabular}

Table 4. Complexity Index for Instance 5
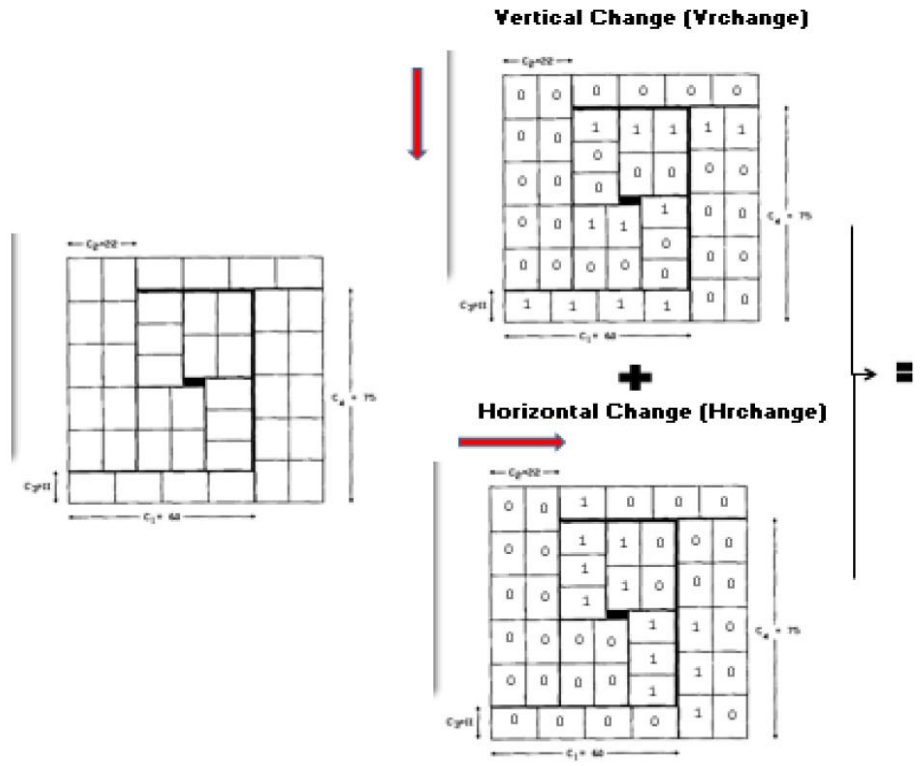

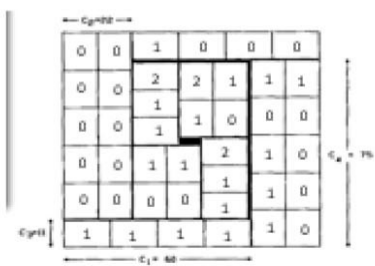

Total Number of changes.
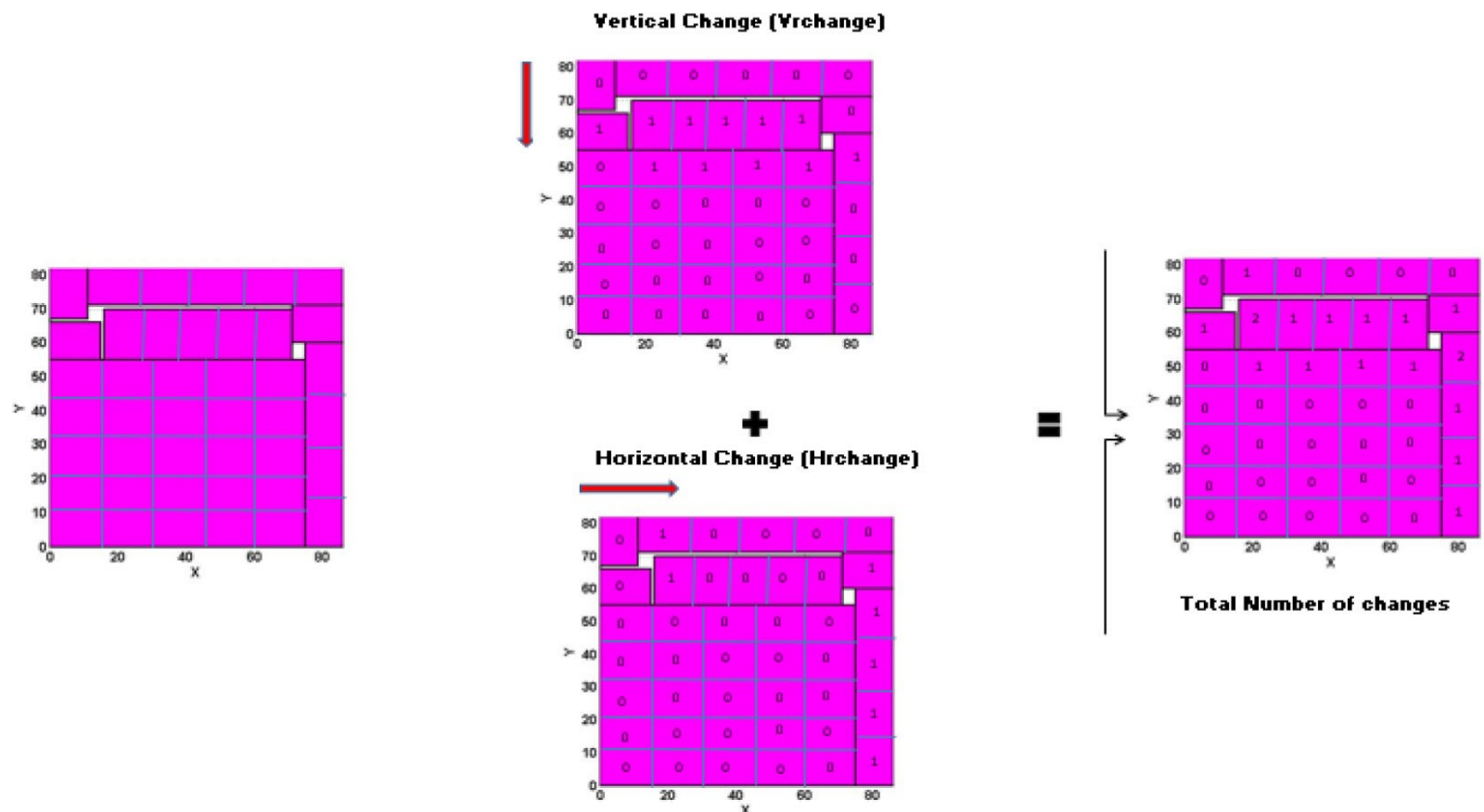

Figure 8. Complexity Index Calculation method for Instance 5

Looking at Instance 18 from Table 2 we can see that relativity a large number of boxes are loaded. The results can be seen in Figure 9 and the Complexity Index comparison is displayed in Table 5. From Figure 9, the literature layout presented in (Scheithauer \& Terno, 1996b) using a G4-heuristic approach is very complex in terms of applying in real-life as it's a time consuming process to change the orientation between most neighbour boxes. While when comparing with the Block layout generated; the number of changes in orientations from one block to the other has been reduced and the boxes that share the same orientation in each Block hold the same orientation. Resulting in a complexity index $\zeta$ for the Block approach of about 0.091 being very close to zero, thus; less 
complex layout compared to the literature complexity index $\zeta$ of 0.97 , which is very close to the value 1 ; meaning, more complex layout.

\begin{tabular}{|l|c|c|c|c|c|c|c|}
\hline Approach & VC & HC & $\boldsymbol{\Sigma} \boldsymbol{B}_{\mathbf{i}}$ & $\mathbf{x r}$ & yc & $\boldsymbol{\zeta}$ & Number of Blocks \\
\hline Block & 15 & 10 & 149 & 15 & 10 & 0.091 & 4 \\
\hline Literature (G4- heuristic approach) & 126 & 140 & 149 & 15 & 9 & 0.97 & 141 \\
\hline
\end{tabular}

Table 5. Complexity Index for Instance 18

\begin{tabular}{|l|l|l|l|l|l|l|l|l|l|l|l|l|l|l|}
\hline 9 & 27 & 29 & 47 & 49 & 67 & 69 & 87 & 89 & 107 & 109 & 127 & 129 & 147 & 149 \\
\hline 8 & 26 & 28 & 46 & 48 & 66 & 68 & 86 & 88 & 106 & 108 & 126 & 128 & 146 & 148 \\
\hline 7 & 23 & 25 & 43 & 45 & 63 & 65 & 83 & 85 & 103 & 105 & 123 & 125 & 143 & 145 \\
\hline & 22 & 24 & 42 & 44 & 62 & 64 & 82 & 84 & 102 & 104 & 122 & 124 & 142 & 144 \\
\hline 6 & 19 & 21 & 39 & 41 & 59 & 61 & 79 & 81 & 99 & 101 & 119 & 121 & 139 & 141 \\
\hline 5 & 18 & 20 & 38 & 40 & 58 & 60 & 78 & 80 & 98 & 100 & 118 & 120 & 138 & 140 \\
\hline 4 & 15 & 17 & 35 & 37 & 55 & 57 & 75 & 77 & 95 & 97 & 115 & 117 & 135 & 137 \\
\hline 3 & 14 & 16 & 34 & 36 & 54 & 56 & 74 & 76 & 94 & 96 & 114 & 116 & 134 & 136 \\
\hline 2 & 11 & 13 & 31 & 33 & 51 & 53 & 71 & 73 & 91 & 93 & 111 & 113 & 131 & 133 \\
\hline 1 & 10 & 12 & 30 & 32 & 50 & 52 & 70 & 72 & 90 & 92 & 110 & 112 & 130 & 132 \\
\hline
\end{tabular}

(a) Literature Layout (Scheithauer \& Terno, 1996b)

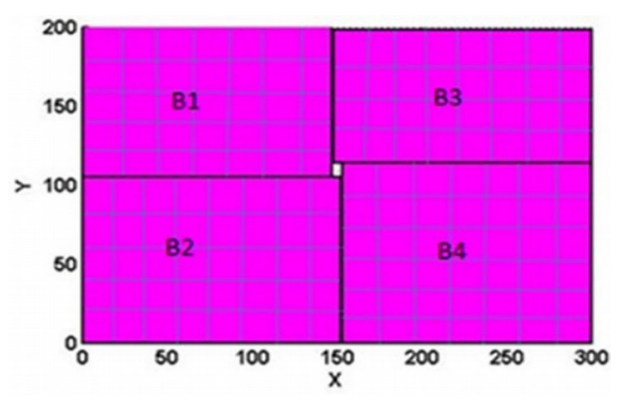

(b) Block Layout

Figure 9. Layouts of Instance 18

As seen from the previous comparisons the alternative layouts proposed by the Block algorithm are much simple in terms of layout complexity while reaching the same optimum solution. Simpler layouts lead to faster loading times as they group the maximum number of boxes along the $\mathrm{X}$ and $\mathrm{Y}$ axis together minimizing the number of rotations required in the space. We believe that such approach will reduce the actual pallet loading time compared to the layout proposed by the above literature papers while minimizing complexity.

\section{Conclusions}

In this paper a (MILP) has been proposed to tackle the Manufacture loading pallet problem known as the (MPLP). The model proposed maximizes the number of boxes loaded on the pallet while grouping boxes that share the same orientation into Blocks. The number of Blocks required can vary from one problem to the other depending on the problem size. The algorithm has been tested against a wide range of datasets and the same optimum results have been obtained, with a very reasonable computational time, ranging from 5 to 1000 seconds for large dataset. Knowing that this problem is classified as NP-hard, such reported computational times are considered acceptable. Also, the algorithm provides the exact geometrical location of the Blocks and provides less complex graphical layouts that outperforms those from literature.

In this paper the main contribution lies in using a linear Block approach that groups boxes of the same orientation across the $\mathrm{X}$ and $\mathrm{Y}$ axis into Blocks, reducing the number of orientation changes within the pallet. Also, a novel Complexity Measure has been presented, that provides a new way to compare 2 pallets of the same size with different graphical layouts. Where a new Index known as $\zeta$ has been introduced and it could hold any value between 0 and 1 describing the complexity of the pallet layout generated by any method. For the datasets tested the Linear Block approach has provided a better complexity index value in addition to the reduction of the number of Blocks used. We believe such reduction will aid the total supply chain operations and a future comparison with time and cost could be implemented to support the claims. 


\section{Declaration of Conflicting Interests}

The authors declared no potential conflicts of interest with respect to the research, authorship, and/or publication of this article.

\section{Funding}

DMA gratefully acknowledges the Saudi Culture Bureau and King Abdulaziz University (KAU) funding.

\section{References}

Alvarez-Valdés, R., Parreño, F., \& Tamarit, J.M. (2005). A branch-and-cut algorithm for the pallet loading problem. Computers \& Operations Research, 32, 3007-3029. https://doi.org/10.1016/j.cor.2004.04.010

Amaral, A.R.S., \& Wright, M. (2001). Experiments with a strategic oscillation algorithm for the pallet loading problem. International Journal of Production Research, 39, 2341-2351. https://doi.org/10.1080/00207540110044589

Arenales, M., \& Morabito, R. (1995). An AND/OR-graph approach to the solution of two-dimensional nonguillotine cutting problems. European Journal of Operational Research, 84, 599-617. https://doi.org/10.1016/03772217(95)00026-M

Arghavani, J., \& Abdou, G. (1996). 3D volumetric pallet-loading optimisation. The International Journal of Advanced Manufacturing Technology, 11, 425-429. https://doi.org/10.1007/BF01178968

Beasley, J.E. (1985). An exact two-dimensional non-guillotine cutting tree search procedure. Operations Research, 33, 49-64. https://doi.org/10.1287/opre.33.1.49

Bhattacharya, S., Roy, R., \& Bhattacharya, S. (1998). An exact depth-first algorithm for the pallet loading problem. European Journal of Operational Research, 110, 610-625. https://doi.org/10.1016/S0377-2217(97)00272-5

Birgin, E.G., Lobato, R.D., \& Morabito, R. (2010). An effective recursive partitioning approach for the packing of identical rectangles in a rectangle. Journal of the Operational Research Society, 61, 306-320.

https://doi.org/10.1057/jors.2008.141

Birgin, E.G., Morabito, R., \& Nishihara, F.H. (2005). A note on an L-approach for solving the manufacturer's pallet loading problem. Journal of the Operational Research Society, 56(12), 1448-1451.

https://doi.org/10.1057/palgrave.jors.2601960

Bischoff, E., \& Dowsland, W.B. (1982). An application of the micro to product design and distribution. Journal of the Operational Research Society, 33, 271-280. https://doi.org/10.1057/jors.1982.54

Castro, P.M., \& Grossmann, I.E. (2012). From time representation in scheduling to the solution of strip packing problems". Computers \& Chemical Engineering, 44, 45-57. https://doi.org/10.1016/j.compchemeng.2012.05.002

Castro, P.M., \& Oliveira, J.F. (2011). Scheduling inspired models for two-dimensional packing problems. European Journal of Operational Research, 215, 45-56. https://doi.org/10.1016/j.ejor.2011.06.001

Dowsland, K.A. (1984). The three-dimensional pallet chart: an analysis of the factors affecting the set of feasible layouts for a class of two-dimensional packing problems. Journal of the Operational Research Society, 35, 895-905. https://doi.org/10.1057/jors.1984.180

Dowsland, K.A. (1987). An exact algorithm for the pallet loading problem. European Journal of Operational Research, 31, 78-84. https://doi.org/10.1016/0377-2217(87)90140-8

Dyckhoff, H. (1990). A typology of cutting and packing problems. European Journal of Operational Research, 44(2), 145-159. https://doi.org/10.1016/0377-2217(90)90350-K

Ji, Y.-G., \& Jin, G.-W. (2009). A New Exact Algorithm Using the Stair Structure for the Pallet Loading Problem. Journal of the Korean Operations Research and Management Science Society, 34, 43-53.

Lau, H.C.W., Chan, T.M., Tsui, W.T., Ho, G.T.S., \& Choy, K.L. (2009). An AI approach for optimizing multi-pallet loading operations. Expert Systems with Applications, 36, 4296-4312. https://doi.org/10.1016/j.eswa.2008.03.024

Letchford, A.N., \& Amaral, A. (2001). Analysis of upper bounds for the pallet loading problem. European Journal of Operational Research, 132(3), 582-593. https://doi.org/10.1016/S0377-2217(00)00163-6 
Lim, A., Ma, H., Xu, J., \& Zhang, X. (2012). An iterated construction approach with dynamic prioritization for solving the container loading problems. Expert Systems with Applications, 39, 4292-4305.

https://doi.org/10.1016/j.eswa.2011.09.103

Lins, L., Lins, S., \& Morabito, R. (2003). An L-approach for packing (1, w)-rectangles into rectangular and L-shaped pieces. Journal of the Operational Research Society, 54, 777-789. https://doi.org/10.1057/palgrave.jors.2601553

Martins, G.H.A., \& Dell, R.F. (2007). The minimum size instance of a pallet loading problem equivalence class. European Journal of Operational Research, 179, 17-26. https://doi.org/10.1016/j.ejor.2006.03.009

Martins, G.H.A., \& Dell, R.F. (2008). Solving the pallet loading problem. European Journal of Operational Research, 184, 429-440. https://doi.org/10.1016/j.ejor.2006.11.012

Morabito, R., \& Morales, S. (1998). A simple and effective recursive procedure for the manufacturer's pallet loading problem. Journal of the Operational Research Society, 49, 819-828. https://doi.org/10.1057/palgrave.jors.2600588

Nelißen, J. (1995). How to use structural constraints to compute an upper bound for the pallet loading problem. European Journal of Operational Research, 84, 662-680. https://doi.org/10.1016/0377-2217(95)00030-T

Ortmann, F.G., Ntene, N., \& Van Vuuren, J.H. (2010). New and improved level heuristics for the rectangular strip packing and variable-sized bin packing problems. European Journal of Operational Research, 203, 306-315. https://doi.org/10.1016/j.ejor.2009.07.024

Papageorgiou, L.G., \& Rotstein, G.E. (1998). Continuous-domain mathematical models for optimal process plant layout. Industrial \& Engineering Chemistry Research, 37, 3631-3639. https://doi.org/10.1021/ie980146v

Pureza, V., \& Morabito, R. (2003). Uma heurística de busca tabu simples para o problema de carregamento de paletes do produtor. Pesquisa Operacional, 23, 359-378. https://doi.org/10.1590/S0101-74382003000200007

Pureza, V., \& Morabito, R. (2006). Some experiments with a simple tabu search algorithm for the manufacturer's pallet loading problem. Computers \& Operations Research, 33, 804-819. https://doi.org/10.1016/j.cor.2004.08.009

Ribeiro, G.M., \& Lorena, L.A.N. (2007). Lagrangean relaxation with clusters and column generation for the manufacturer's pallet loading problem. Computers \& Operations Research, 34(9), 2695-2708. https://doi.org/10.1016/j.cor.2005.10.008

Scheithauer, G., \& Terno, J. (1996a). A beuristic approach for solving the multi-pallet packing problem. Dresden University.

Scheithauer, G., \& Terno, J. (1996b). The G4-heuristic for the pallet loading problem. Journal of the Operational Research Society, 47, 511-522. https://doi.org/10.1057/jors.1996.57

Silva, E., Oliveira, J.F., \& Waescher, G. (2016). The pallet loading problem: a review of solution methods and computational experiments. International Transactions in Operational Research, 23(1-2), 147-172. https://doi.org/10.1111/itor.12099

Tsai, R.D., Malstrom, E.M., \& Kuo, W. (1993). Three dimensional palletization of mixed box sizes. IIE Transactions, 25, 64-75. https://doi.org/10.1080/07408179308964305

Wäscher, G., Haußner, H., \& Schumann, H. (2007). An improved typology of cutting and packing problems. European Journal of Operational Research, 183, 1109-1130. https://doi.org/10.1016/j.ejor.2005.12.047

Wei, L., Zhang, D., \& Chen, Q. (2009). A least wasted first heuristic algorithm for the rectangular packing problem. Computers \& Operations Research, 36, 1608-1614. https://doi.org/10.1016/j.cor.2008.03.004

Westerlund, J., \& Papageorgiou, L.G. (2004). Improved performance in process plant layout problems using symmetry-breaking constraints. Discovery through Product and Process Design, Proceedings of FOCAPD (485-488).

Wu, K.C., \& Ting, C.J. (2007). A Two-phase algorithm for the manufacturer's pallet loading problem. Industrial Engineering and Engineering Management, 2007 IEEE International Conference On (1574-1578).

https://doi.org/10.1109/IEEM.2007.4419457

Young-Gun, G., \& Kang, M.-K. (2001). A fast algorithm for two-dimensional pallet loading problems of large size. European Journal of Operational Research, 134, 193-202. https:/ /doi.org/10.1016/S0377-2217(00)00249-6 


\section{Appendix A}

Additional graphical layouts

Additional graphical layouts for the proposed complexity measure are presented in this section, the tests are performed using the datasets presented in Table 2:

\section{Instance 14:}

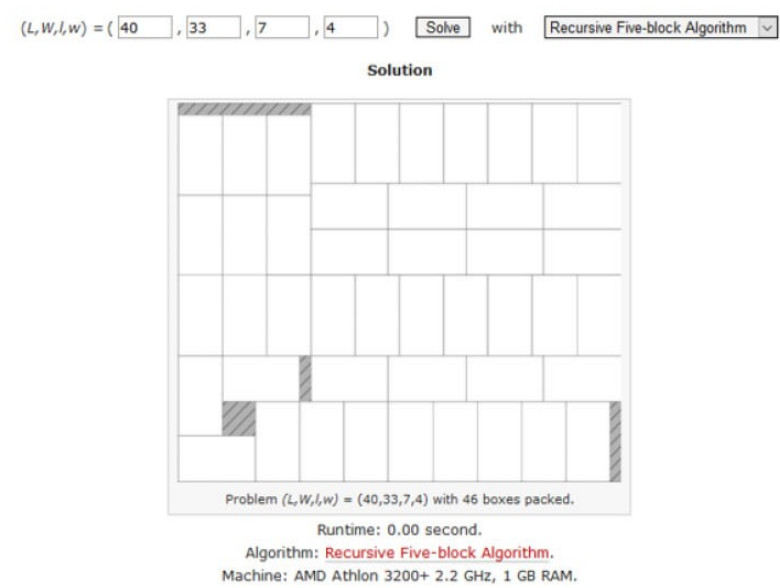

(a) Literature Layout (Birgin et al., 2010)

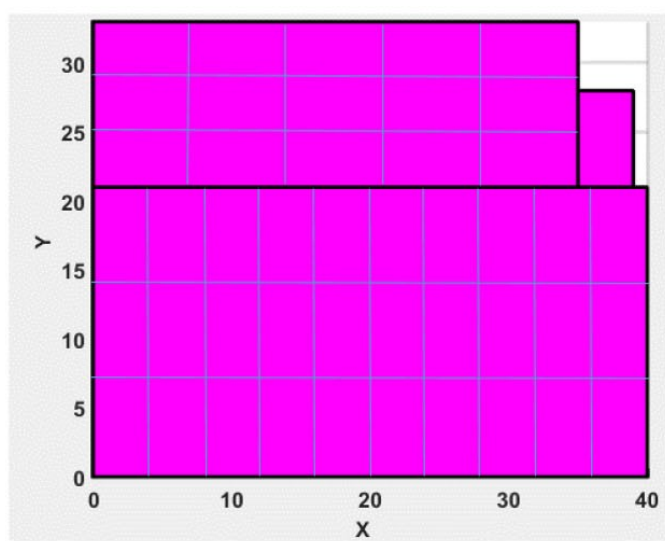

(b) Block Layout

\begin{tabular}{|l|c|c|c|c|c|c|c|}
\hline Approach & VC & HC & $\boldsymbol{\Sigma} \boldsymbol{B}_{\mathrm{i}}$ & $\mathbf{x r}$ & yc & $\boldsymbol{\zeta}$ & Number of Blocks \\
\hline Block & 9 & 1 & 46 & 5 & 6 & 0.123 & 3 \\
\hline Literature (Recursive 5-block approach) & 25 & 4 & 46 & 10 & 5 & 0.376 & 9 \\
\hline
\end{tabular}

\section{Instance 17:}

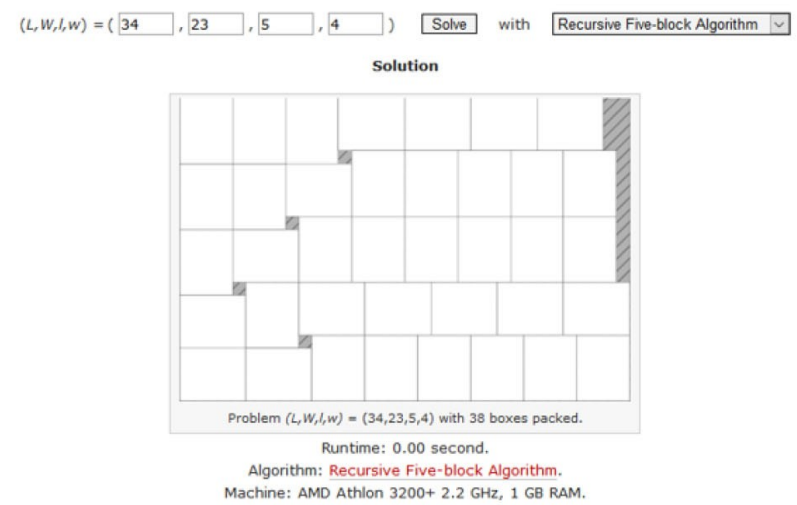

(a) Literature Layout (Birgin et al., 2010)

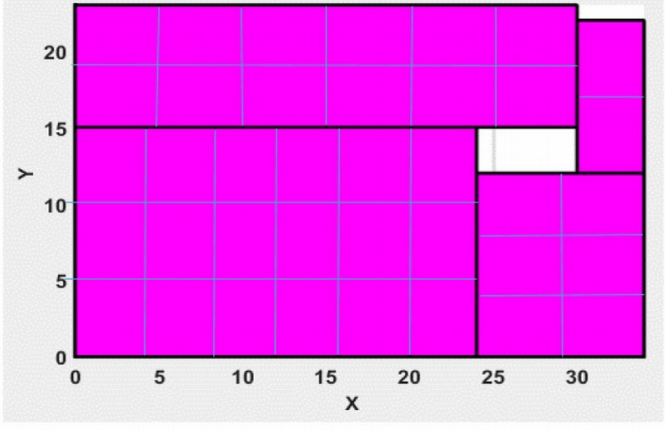

(b) Block Layout

\begin{tabular}{|l|c|c|c|c|c|c|c|}
\hline Approach & VC & HC & $\boldsymbol{\Sigma} \boldsymbol{B}_{\mathrm{i}}$ & $\mathbf{x r}$ & yc & $\boldsymbol{\zeta}$ & Number of Blocks \\
\hline Block & 7 & 5 & 38 & 7 & 5 & 0.187 & 4 \\
\hline Literature (Recursive 5-block approach) & 22 & 8 & 38 & 7 & 5 & 0.468 & 13 \\
\hline
\end{tabular}




\section{Instance 21:}
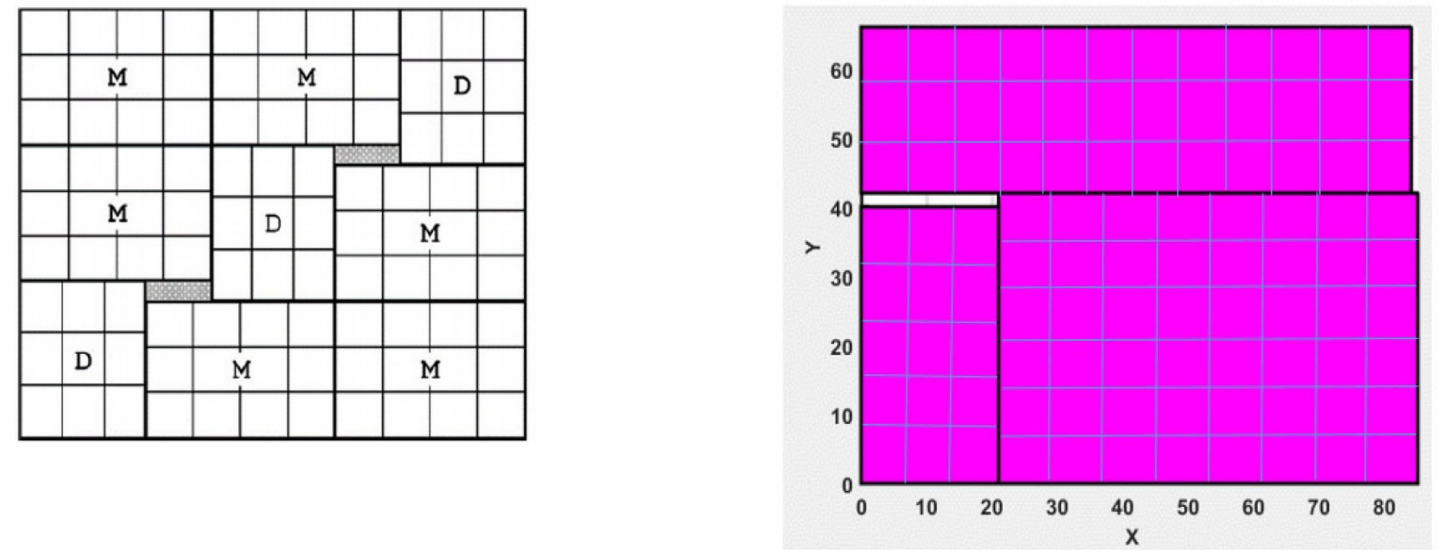

(a) Literature Layout (Martins \& Dell, 2008)

(b) Block Layout

\begin{tabular}{|l|c|c|c|c|c|c|c|}
\hline Approach & VC & HC & $\boldsymbol{\Sigma} \boldsymbol{B}_{\mathrm{i}}$ & $\mathbf{x r}$ & yc & $\boldsymbol{\zeta}$ & Number of Blocks \\
\hline Block & 8 & 6 & 99 & 12 & 8 & 0.078 & 3 \\
\hline Literature (HVZ algorithm) & 13 & 12 & 99 & 11 & 9 & 0.14 & 13 \\
\hline
\end{tabular}

Instance 51:

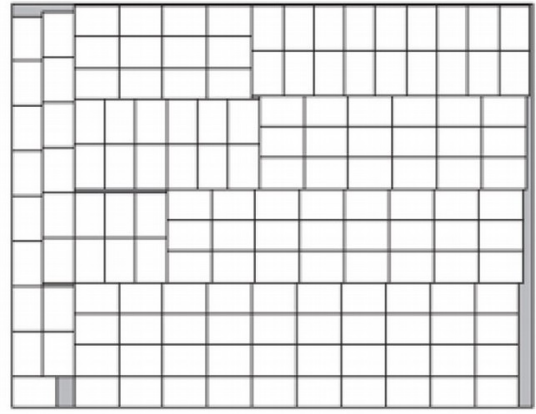

Instance $(1600,1230,137,95)$ with 147 boxes

(a) Literature Layout (Birgin et al., 2010)

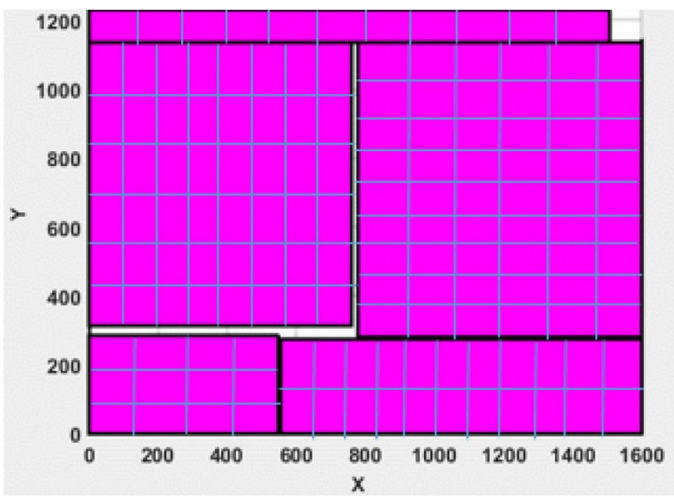

(b) Block Layout

\begin{tabular}{|l|c|c|c|c|c|c|c|}
\hline Approach & VC & HC & $\boldsymbol{\Sigma} \boldsymbol{B}_{\mathrm{i}}$ & $\mathbf{x r}$ & yc & $\boldsymbol{\zeta}$ & Number of Blocks \\
\hline Block & 23 & 11 & 147 & 11 & 10 & 0.12 & 5 \\
\hline Literature (Recursive 5-block approach) & 26 & 15 & 147 & 15 & 9 & 0.15 & 11 \\
\hline
\end{tabular}

Journal of Industrial Engineering and Management, 2021 (www.jiem.org)

\section{(c) (1) ( $)$}

Article's contents are provided on an Attribution-Non Commercial 4.0 Creative commons International License. Readers are allowed to copy, distribute and communicate article's contents, provided the author's and Journal of Industrial Engineering and Management's names are included. It must not be used for commercial purposes. To see the complete license contents, please visit https://creativecommons.org/licenses/by-nc/4.0/. 